\title{
La fuerza de las mujeres: un estudio de las estrategias de resiliencia y la transformación en la ocupación humana de mujeres víctimas del conflicto armado en Colombia ${ }^{1}$
}

\author{
Women's strength: a study on resilience strategies and transformation \\ in the human occupation of women victims of the armed conflict in \\ Colombia
}

\author{
Luz Ángela Albarracín Cerquera² \\ Karol Andrea Contreras Torres ${ }^{3}$ \\ Recibido: 27 de febrero 2017 • Enviado para modificación: 24 de junio 2017 • Aceptado: 11 de agosto 2017 \\ Albarracín, L.A. \& Contretas, K. (2017). La fuerza de las mujeres: un estudio de las estrategias de resiliencia y \\ la transformación en la ocupación humana de mujeres víctimas del conflicto armado en Colombia. Revista \\ Ocupación Humana, 17 (1), 25-38.
}

\section{RESUMEN}

La investigación tuvo por objetivo identificar estrategias de resiliencia frente a la transformación de la ocupación humana en mujeres víctimas de desplazamiento forzado en el marco del conflicto armado colombiano. Se definen los conceptos de resiliencia, ocupación humana, cuerpo y conflicto armado, y hechos victimizantes, diferenciados hacia las mujeres, así como sus consecuencias en la transformación de las actividades significativas. Se realiza un estudio cualitativo en el que participan cuatro mujeres mayores de 18 años, residentes en Bogotá y víctimas de desplazamiento forzado. Se emplean entrevistas semi-estructuradas e indagación corporal mediante elementos de danza orgánica, danza butoh y danza contacto. Los datos fueron analizados a la luz de categorías de ocupación y resiliencia previamente establecidas. Las principales estrategias de resiliencia de estas mujeres están relacionadas con el hacer por los otros; el autoconocimiento y la autogestión en el restablecimiento de sus proyectos de vida, y la espiritualidad, desde la conciencia de sus fortalezas para hacer frente al día a día. Se observa una búsqueda activa de nuevos espacios de encuentro per-

\footnotetext{
1Artículo derivado de la investigación La Fuerza de las Mujeres: un Estudio de las Estrategias de Resiliencia y la Transformación en la Ocupación Humana de Mujeres Víctimas del Conflicto Armado en Colombia, realizada por las autoras en 2016 en el marco de la Especialización en Desarrollo Humano con Énfasis en Procesos Afectivos y Creatividad de la Universidad Distrital Francisco José de Caldas.

${ }^{2}$ Terapeuta Ocupacional. Especialista en Desarrollo Humano con Énfasis en Procesos Afectivos y Creatividad. Fundación Art Suigeneris. Bogotá, Colombia. angelaac87@hotmail.com

${ }^{3}$ Psicóloga. Especialista en Desarrollo Humano con Énfasis en Procesos Afectivos y Creatividad. Bogotá, Colombia. Corporación Mujeres Transformando. karolacontrerast@gmail.com
} 
sonal, de reconocimiento de sus capacidades y potencialidades a partir de la formación, el disfrute e interacción con otros y la productividad.

\title{
PALABRAS CLAVE
}

Conflicto armado, persona desplazada, víctima de guerra, mujer, expresión corporal

\begin{abstract}
The research aimed to identify strategies of resilience against the transformation of human occupation in women who are victims of forced displacement, in the context of the Colombian armed conflict. Concepts defined here are resilience, human occupation, body and armed conflict, and victimizing events, characterized to women, and their consequences in the transformation of meaningful activities. A qualitative study is carried out, in which the participants were four women over the age of 18, living in Bogotá and victims of forced displacement. In addition, semi-structured interviews were conducted and body language (dance) is investigated, through elements of organic dance, Butoh dance and Contact improvisation. The data was analyzed in the light of previously established occupation and resilience categories. The main resilience strategies of these women are related to "doing for others"; self-knowledge and self-management in the restoration of their life projects, and spirituality, from the awareness of their strengths to face the day to day. There is an active search for new spaces of personal encounter, recognition of their capabilities and potential, derived from training, enjoyment and interaction with others, and from productivity.
\end{abstract}

\section{KEY WORKS}

Armed conflict, displaced persons, war victims, woman, corporal expression

\section{Introducción}

Colombia, desde hace más de 50 años, vive un continuo de violencias sociopolíticas que han afectado directamente y de forma diferencial a la población civil, de acuerdo al nivel de vulnerabilidad en que se encuentren. A la luz de esta realidad y en el escenario de postconflicto, se requiere conocer las estrategias de resiliencia que estas personas han desarrollado frente a la transformación de sus actividades significativas ante situaciones de adversidad, en aras de aportar a la reparación del tejido social.

De acuerdo con Fores y Grané (2011), para la conceptualización de la resiliencia se deben comprender sus dos nociones básicas: adversidad, entendida como el trauma, riesgo o amenaza presente en la vida de una persona como una herida que la aproxima a la muerte; y la adaptación positiva, entendida como la superación del trauma. En este sentido, Cyrulnik (2007) afirma que para "resiliar" una situación de malestar hace falta que la persona haya sido vulnerada, herida, traumatizada, fracturada o desgarrada, es decir, que haya sufrido tal magnitud de lesión para que se dé la transformación de su vida. De esta forma, se asume que la resiliencia es la capacidad de cualquier ser humano para reconocer 
y usar sus propios recursos y los que le provea el medio para hacer frente a una situación de adversidad. Desde la experiencia de las investigadoras en la atención a víctimas, se reconoce que la resiliencia se revela de diferentes maneras, entre ellas se definen las siguientes categorías, que son las que se emplean en esta investigación.

Agenciamiento: Yo para mí. Acciones que propende y realiza la persona por sí misma, sin presión ni refuerzo de otras, sino que considera debe hacer para su propio bienestar. Entre estas se observan acciones de autoconcepto, autovaloración, hacer por otros y otras, la capacidad de perdón, el disfrute de la sexualidad y la reflexividad.

VINCULACIÓN afECTIVA: De los otros para mí. Acciones cuya procedencia está en los otros y son percibidas como de bienestar para la persona. Se relaciona con el establecimiento de sanas relaciones interpersonales, de apropiadas redes de apoyo y predisponentes en los sistemas de creencias y las prácticas culturales del contexto.

Acciones del Estado: El Estado para mí. Acciones ordenadas por la jurisprudencia del Estado que tienen como fin dar trámite y atención a las situaciones de adversidad en el marco del conflicto armado. Para ello se hace hincapié en la percepción de justicia y en la mirada crítica frente a las acciones estatales.

ENTORNO PARA mí: Se define como la influencia que tiene el entorno en el desarrollo o fortalecimiento de las estrategias de resiliencia. Da cuenta de las redes de apoyo que pueden facilitar el proceso y de cómo el contexto influ- ye directamente en el proceso resiliente. De igual manera, para esta categoría se determina la ampliación de redes, lo cual favorece la participación en otros entornos y la adquisición de nuevos roles ocupacionales; se valida la voz de las mujeres y se promueven espacios de disfrute como eje fundamental en el desarrollo de actividades con sentido y significado para ellas.

EsPIRITUALIDAD: Si bien esta categoría no se explora directamente, se reconoce como la vinculación afectiva de las mujeres con una fuerza interior que les permite hacer frente a las situaciones de adversidad. Se manifiesta como reconocimiento de una fuerza innata, la vinculación con un ser supremo o el arraigo a la vida. La espiritualidad, según Castañeda (2002), implica tener cada día una razón para vivir, para humanizarse e intentar alcanzar los sueños; aquella capacidad propia que tiene el ser humano de tomar conciencia de su fortaleza para superarse día tras día sin desfallecer.

De otro lado, la ocupación humana se entiende como uno de los ejes centrales del ser en sus distintas dimensiones. De acuerdo con el Marco de Trabajo para la Terapia Ocupacional de la Asociación Americana de Terapia Ocupacional (AOTA, 2008/2010), se refiere a diversas áreas del desempeño que determinan aspectos fundamentales del ser humano para desarrollarse en los diferentes entornos. Tales áreas se definen de la siguiente manera: actividades de la vida diaria, que en este documento se denominan actividades básicas o de supervivencia; actividades instrumentales de la vida diaria, denominadas potenciamiento o actividades más estructuradas; ocio y tiempo libre, 
que corresponden a la categoría de disfrute; por último, el área de participación social o comunitaria.

Las actividades cotidianas -básicas o de supervivencia- están orientadas al cuidado del propio cuerpo (Rogers y Holm, citados en AOTA, 2008/2010). También se refieren a las actividades básicas de la vida diaria, son fundamentales para vivir en un mundo social y permiten la supervivencia y el bienestar (Christiasen y Hammecker, citados en AOTA, 2008/2010). Las actividades de la vida diaria contemplan la realización de tareas como dormir, descansar, el acicalado, la alimentación y el baño. La ejecución de las mismas promueve el bienestar, el cuidado de sí mismo y la resignificación en la vida, ya que aumentan la autoestima, la motivación y el autoconcepto.

Las actividades instrumentales de la vida diaria o actividades más estructuradas hacen referencia a actividades de apoyo a la vida cotidiana en la casa y la comunidad; requieren interacciones más complejas, en comparación con las que se utilizan en las actividades de la vida diaria. Dentro de ellas se incluyen el cuidado de otros y el cuidado de los hijos. Estas actividades se consideran más complejas ya que requieren e involucran a otros; su desarrollo permite la construcción del proyecto de vida, determinado por aspectos relacionados con la motivación intrínseca para la participación, la habituación y la ejecución de roles ocupacionales a partir de los propios intereses y el funcionamiento volitivo. Incluyen la educación y el trabajo, actividades y ejes importantes para las mujeres víctimas del conflicto armado.
La participación social o comunitaria alude a patrones de comportamiento organizados que son característicos y esperados de un individuo dentro de un sistema social (Mosey, citada en AOTA, 2008/2010). Se centran en la participación en la comunidad, la cual busca la interacción en el barrio, con los vecinos y las organizaciones sociales, teniendo en cuenta el contexto de las mujeres víctimas.

El ocio, tiempo libre o actividades de disfrute hace referencia a actividades no obligatorias, intrínsecamente motivadas y en las cuales se participa durante un tiempo discrecional o libre, es decir, un tiempo no comprometido con ocupaciones obligatorias tales como el trabajo o el cuidado propio (Parham y Fazio, citados en AOTA, 2008/2010). Incluye actividades de exploración y participación en el ocio.

Ligar las categorías de resiliencia y ocupación humana a las realidades de la guerra implica reconocer primero el conflicto armado colombiano como un conflicto interno, teniendo en cuenta que sus raíces son motivaciones domésticas y no de magnitud internacional, razón por la cual se perpetúa dentro de las fronteras del Estado. Así, se entiende como el enfrentamiento de diferentes grupos armados que utilizan como estrategias la amenaza, la violación de los derechos humanos y la protección como búsqueda para incrementar su poder sobre la población civil. Involucra a diferentes actores: las guerrillas, los grupos paramilitares y las fuerzas del Estado (Salazar y Castillo, citado por Valencia, 2006; Trejos, 2013).

Por otro lado, se requiere introducir las realidades de la estructura so- 
cio-cultural y política que han facilitado el sostenimiento de una violación sistemática de los derechos de las mujeres en el país. La violencia hacia la mujer se define, según la Ley 1257 de 2008 (Congreso de Colombia, 2008), como cualquier acción u omisión que le cause muerte, daño o sufrimiento físico, sea este sexual, psicológico, económico o patrimonial, por su condición de mujer, así como las amenazas de tales actos, la coacción o la privación arbitraria de la libertad, bien sea que se presente en el ámbito público o privado. Esto implica que la violencia hacia las mujeres en el marco del conflicto armado es también una violencia basada en el género, pues está soportada en prácticas y preconcepciones socioculturales acerca de la desigualdad de las mujeres.

El Auto 092 de 2008 de la Corte Constitucional refiere:

"la violencia ejercida en el conflicto armado interno colombiano victimiza de manera diferencial y agudizada a las mujeres; por su condición de género están expuestas a riesgos particulares y vulnerabilidades específicas (...), que a su vez son causas de desplazamiento, y por lo mismo, explican en su conjunto el impacto desproporcionado; como víctimas sobrevivientes de actos violentos, se ven forzadas a asumir roles familiares, económicos y sociales distintos a los acostumbrados. Las mujeres deben sobrellevar cargas materiales y psicológicas de naturaleza extrema y abrupta, que no afectan de igual manera a los hombres."

(Corte Constitucional, 2008)
De este modo, la guerra produce desarraigos y malestares emocionales que se traducen en los lenguajes del cuerpo. Dando soporte a esta postura, María del Carmen Castañeda (2011) enuncia el cuerpo como un lugar simbólico de comunicación y de escritura en donde se registran representaciones, imágenes y metáforas que evocan más de lo que en realidad se dice. La narración sobre los cuerpos puede revelar historias colectivas que constituyen prácticas del existir humano en tanto sus emocionalidades, comportamientos, creencias. Desde la noción de guerra, vemos que el cuerpo no es solo carne, también es un escenario de relaciones de poder, es un texto donde se inscribe y naturaliza lo político (Campero, 2011).

A partir de esta comprensión del cuerpo como instrumento de lenguaje y de interrelación con el mundo, se realiza un acercamiento a la danza como vía de indagación, no solamente como movimiento corporal acompañado por música, sino como producciones discursivas a través de "performances emocionales" (Belli, Rom \& Lupicinio, citados por Muntanyola-Saura \& Belli, 2014).

Siguiendo la percepción de Britto (2009), las mujeres no solo son víctimas en tanto madres, esposas e hijas, sino que sus cuerpos han sido también campo de batalla; por ello, la preservación de la memoria se hace una tarea dolorosa, pero puede ser también una forma de conjurar el pasado trágico y redefinir un proyecto de vida. Lo anterior pone en evidencia la necesidad de buscar estrategias para superar las repercusiones de estos hechos y las implicaciones que esto tiene sobre la 
ocupación humana.

Las danzas seleccionadas para identificar estas estrategias de resiliencia fueron: danza orgánica, danza contacto y danza butoh. La danza orgánica o no movimiento parte de comprender que el ser humano tiene códigos de lenguaje no verbal transmitidos a través de sensaciones, emociones que emite cada uno de los órganos vitales. Toma como eje central la postura de Pina Bausch, para quien cobran particular importancia la gestualidad, los sonidos, el silencio prolongado, los gritos, las risas, la respiración desesperada, la palabra que evoca; tiene en cuenta el expresionismo a partir de la época de la postguerra y agrega que "incluso las palabras solo pueden evocar cosas, ahí es donde la danza entra de nuevo" (Bausch, citada por Quiroga, 2012, p.4). De esta manera, la danza orgánica permite identificar el encuentro consigo mismo, el Yo para mí donde la mujer víctima del conflicto armado realiza un autoreconocimiento desde la subjetividad de su ser.

Entre tanto, la danza contacto provee experiencias perceptuales de los cuerpos externos en relación con la captación de experiencias internas, las cuales se manifiestan en la interacción del propio cuerpo con las fuerzas físicas de otros que lo afectan en todo momento. Además, ofrece el surgimiento de una fuerza creada por la interacción cooperativa entre las bailarinas a partir de los sentidos del tacto y kinestésico, que es definida por el equilibrio de las inercias, psicologías y espíritus de los participantes, entre quienes se desarrolla una conversación táctil. La danza contacto, a diferencia de otros tipos, plantea una continuidad más extrema entre la danza y la vida cotidiana, y además permite acercar las zonas tabú de la interacción social, dado que propone un modo de interacción íntimo y sensitivo entre individuos sin importar sus diferencias y sin un valor sexual o de confrontación (Menacho, 2008). Para esta investigación, la danza contacto permite evidenciar desde el cuerpo los estados de resiliencia frente a los otros para mí y el entorno para mí, a través de las actividades significativas de participación comunitaria.

En cuanto a los elementos de danza butoh, en la investigación se utilizan la relación cuerpo y mente y la relación con los opuestos, que en este caso tienen que ver con aspectos de malestar vividos por las mujeres víctimas, haciendo un paralelo con sus dinámicas de vida y su situación actual. Teóricamente, se entiende que "el cuerpo en el Butoh es el Butoh-tai, muy difícil de traducir pero al que nos podemos aproximar a través de una actitud física y mental que logre integrar los elementos dicotómicos del ser humano, del funcionamiento de la lógica, de esa necesidad que tiene el cerebro, la mente, de discernir, seleccionar, elegir entre conceptos opuestos (danza-teatro, bueno-malo, viejo-joven, hombre mujer, blanco-negro). En la dicotomía, el discernimiento, la mente elige, y de los aspectos opuestos selecciona uno" (Soler, 2008, p.13). La exploración se centró en los cambios que han presentado las mujeres en la ciudad, desde la influencia que han tenido el Estado y el entorno en su proceso.

A través de las técnicas descritas anteriormente, esta investigación buscó identificar las estrategias de resi- 
liencia frente a la transformación de la ocupación en mujeres víctimas de desplazamiento forzado por cuenta del conflicto armado colombiano.

\section{Método}

En el estudio realizado en la ciudad de Bogotá participaron cuatro mujeres mayores de 18 años, víctimas de desplazamiento forzado por cuenta del conflicto armado, procedentes de los departamentos de Nariño, Tolima y Cundinamarca; su permanencia en la ciudad oscila entre 3 meses y 10 años. Se utilizó un diseño de investigación cualitativo y como instrumentos se emplearon: entrevistas semi estructuradas con base en los constructos adoptados de resiliencia y ocupación humana, e indagación corporal a través de elementos de la danza orgánica, danza butoh y danza contacto.

Se llevaron a cabo cuatro etapas de recolección de datos. En la primera se realizó la entrevista semiestructurada a cada una de las participantes, en las siguientes tres la indagación a partir de elementos de danza. Se inició por la danza orgánica, para explorar la categoría yo para mí, en relación con las actividades diarias y estructuradas. Posteriormente la danza butoh, para la indagación de las categorías Estado para mí y otros para mí, en relación al disfrute. Finalmente, la danza contacto, para analizar las categorías otros para mí y entorno para mí, en relación con la participación comunitaria. Se realizó análisis categorial de la información recolectada a partir de una matriz de cruce de categorías diseñada para tal fin.
Previo a la toma de datos se hizo lectura de un consentimiento informado en el que se especifican los fines académicos de la investigación y la confidencialidad de sus datos personales, despejando dudas y realizando la respectiva aprobación del mismo. A partir de esto se clarifica a las participantes que el objetivo de la investigación no está centrado en la narrativa específica de los eventos victimizantes, al contrario, se propende por un abordaje con una perspectiva proyectiva desde aspectos resilientes.

\section{Resultados y discusión}

\section{Transformación de la ocupación humana}

A partir de la indagación fue evidente que las mujeres participantes han vivido diferentes hechos victimizantes, no solo han sufrido el desplazamiento, también delitos contra la integridad sexual, torturas, amenazas, homicidios y desapariciones forzadas. Esto conlleva situaciones de adversidad que se relacionan con la transformación de sus ocupaciones en la vida cotidiana, entendiendo que ocuparse es importante para las personas de todas las edades y que ello contribuye al estar-bien individual, al progreso y a la equidad social (Grupo de Investigación Ocupación y Realización Humana, 2011). Dichas transformaciones se evidenciaron, según sus narrativas, en la afectación de sus roles cotidianos en razón al cambio hostil y abrupto del contexto, el cual no les garantiza los mínimos para una adecuada adaptación.

Tales cambios pueden variar de acuerdo al tiempo de permanencia en 
la ciudad, lo que se reflejaba en sus estrategias de resiliencia y en la ejecución de actividades ocupacionales. Se identificó una mayor dificultad en el proceso de reestructuración del proyecto de vida en las participantes que llevaban poco tiempo en la ciudad, sumado a que sus vínculos afectivos más significativos permanecían en el municipio expulsor; esto genera una carga emocional adicional, dados los cambios en las dinámicas de sus roles.

En relación con la transformación en la participación comunitaria, esta se puede potenciar o limitar de acuerdo a las características o facilitadores del entorno, que pueden ser particulares al rol que desempeñen dentro de las estructuras formales de organización comunitaria. A las limitaciones en la participación comunitaria se suma un malestar emocional relacionado con la culpa, el duelo y la impotencia ante la imposibilidad de continuar haciendo parte de acciones cooperativas para poblaciones vulnerables, esto implica que su posición de liderazgo se convierta en vulnerabilidad como consecuencia del desplazamiento forzado. Las participantes que han permanecido por un periodo prolongado en la ciudad identificaron mayores oportunidades para la participación comunitaria, desde la validación de su voz en los diferentes espacios que les ofrece el contexto; esto redunda en su bienestar y en el empoderamiento que se deriva del reconocimiento de sus derechos, potencialidades y recursos propios.

Finalmente, en la transformación de los roles ocupacionales relacionados con actividades de disfrute, se evidenció que estas mujeres han reconocido su derecho a la participación en actividades de ocio y tiempo libre, incluso antes del desplazamiento. Sin embargo, las dinámicas de estas actividades se han visto afectadas por aspectos contextuales como la distancia y las posibilidades de acceso. Uno de los aspectos fundamentales que media la participación en estos espacios es el recurso económico que demandan; mientras en sus lugares de origen tenían alternativas de disfrute y ocio que no significaban gastos, como ir al río, participar de eventos locales, entre otros; en la ciudad ir a los parques, cines, presentaciones $u$ otras actividades, no solo implica gastos de desplazamiento, también de alimentación y de acceso. La participación en actividades de disfrute se centra más en su núcleo familiar que en ellas mismas, en razón a una búsqueda de bienestar para sus allegados que compense las pérdidas generadas por el conflicto armado.

Las transformaciones en la ocupación humana de las mujeres participantes contrastan con las comprensiones del Grupo de Trabajo Mujer y Género, por la Verdad, la Justicia, la Reparación y la Reconciliación (2008), quienes consideran que en el conflicto armado el cuerpo y la dignidad de ser mujer son especialmente vulnerados, junto al menosprecio por ella, también se desea someterla, demostrar el poder de unos sobre otros: de los hombres sobre las mujeres y de los hombres de un bando sobre las mujeres que se encuentran en el otro. El grupo afirma que se usa a la mujer y a su cuerpo como arma de guerra para humillar al enemigo, disponiendo de este y de su dignidad para señalar que se puede ser "dueño" de la vida y del placer o alegrías que ellas pueden dar; se actúa de manera arbitraria como omnímodos dueños con un 
territorio. En períodos de conflicto armado, los antivalores y las tendencias más primitivas y violentas del hombre como género se permiten, se toleran y reciben un permiso tácito de usar lo femenino al arbitrio de eso masculino más primitivo. Por eso, la violación de los derechos de las mujeres en las guerras y los conflictos armados tiene una connotación aún más brutal que en periodos diferentes.

\section{Identificación de estrategias de resiliencia}

\section{Categoría Yo para mí - Otros para mí.} Ante las situaciones de adversidad vividas, las mujeres participantes han generado diferentes estrategias. Si bien hay un ejercicio activo en las diferentes categorías de resiliencia (yo para mí, otros para mí, entorno para mí y Estado para mí), este se enfoca en las categorías otros para mí y yo para mí. De esta manera, manifestaron que su principal fuente de motivación es la vinculación afectiva con sus hijos, encontrándose narrativas como: "imis hijos, mi mayor motivación!", "mis hijos, el motor de mi vida, por ellos todo...", lo cual muestra que sus acciones resilientes están encaminadas al desarrollo de actividades para sus hijos/as. De lo anterior se desprende que sus roles, como ser madre, ser trabajadora y ser proveedora, son el eje fundamental para la reconstrucción de su proyecto de vida en la ciudad.

Dentro de esta categoría se evidencia además que el tiempo de permanencia en la ciudad puede influir. El sentimiento por el cuidado de los otros se magnifica cuando hay una mayor urgencia de satisfacer las necesidades básicas de las personas a cargo, ocasionando que se centren exclusivamen- te en el cuidado de sus hijos/as; esta estrategia es más evidente justo en el momento de llegada a la ciudad. Los otros facilitan el proceso resiliente, el facilitador es la realimentación que reciben de la satisfacción de sus hijos/ as por las acciones que ellas realizan, lo cual funciona como un reforzador de su agenciamiento en la ciudad que les permite tener la percepción de que "después que estén mis hijos bien, yo estoy bien".

En contraste, se evidenció que en la priorización de la estrategia resiliente de otros para mí se han dejado en segundo plano otros roles como ser amiga, ser estudiante y ser pareja. Aun así, la categoría del yo para mí también juega un papel fundamental en la resiliencia de las mujeres, en tanto se manifiesta en su rol de ser mujer a partir de prácticas de autocuidado y de reconocimiento de potencialidades, herramientas y recursos propios, referidas en expresiones como: "mi misión... dar un ejemplo de vida... de ser una mujer trabajadora, verraca y echada para delante... esa es mi misión... que saco a mis hijos adelante, siempre mis hijos por delante...", "lo guerreras, o sea, nunca nos dejamos...", "lo verracas para salir... nunca nos dejamos vencer por ninguna circunstancia", "yo que soy una persona guerrera, trabajadora", "ganas de vivir"; lo cual muestra que identifican su potencialidad de ser mujer en la ciudad a partir de atribuciones de fuerza y arraigo a la vida.

Por otra parte, en esta categoría se encuentran sus metas y sueños establecidos, como factores que siguen latentes a pesar de la adversidad. Estos conllevan la reconstrucción de sus proyectos de vida, principalmen- 
te relacionados con acciones para sí mismas como estudiar, emprender unidades productivas y tener acceso a vivienda propia, las cuales hacen parte de los procesos de reparación y mitigación del daño que se desarrollan en el país ante las consecuencias del conflicto armado.

Marcela Lagarde (2004) menciona que el desarrollo del cuidado propio y de los otros se configura en la vida de las mujeres como un sincretismo de género donde "la cultura patriarcal fomenta en las mujeres la satisfacción del deber de cuidar, convertido en deber ser ahistórico natural de las mujeres $y$, por tanto, deseo propio $y$, al mismo tiempo, la necesidad social y económica de participar en procesos educativos, laborales y políticos" (p.157); de ahí que el uso del tiempo y la energía se invierta prioritariamente en los otros, más que en ellas mismas, encontrando que esa inversión se traduce en una retroalimentación del deber cumplido y de retribución afectiva del cuidado.

Categoría entorno para mí. Dentro de esta categoría se encontró que existen algunos facilitadores que permiten el proceso resiliente, los cuales son determinados a partir de la red de apoyo con que se cuente durante la llegada y la permanencia en la ciudad, como redes institucionales, sociales, familiares, entre otras. Otro facilitador de este proceso es el reconocimiento del entorno, el cual provee una percepción de seguridad y mayor desenvolvimiento en la ciudad.

Las oportunidades que provee el contexto también son determinantes en la reestructuración de la ocupación humana posterior a la adversidad, en razón a que favorecen el desarrollo de actividades que dan sentido y significado a sus vidas. Esto se manifestó en las referencias de las mujeres a la ciudad como un entorno con mejores posibilidades de acceso para ellas y los suyos en términos de salud, educación, trabajo y actividades de disfrute, oportunidades que hacen parte de los ejercicios de restitución de derechos y que se reflejan en la interacción con el medio.

Como consecuencia de las oportunidades que provee el entorno, las mujeres identificaron que este valida sus habilidades y facilita que las mismas sean reconocidas y potenciadas. Por ejemplo, la costura o el arte pueden ser vistos no solo como actividades domésticas y de ocio, sino con capacidad de transformar su ocupación humana, comprendiéndolas como estrategias de productividad y de construcción de sociedad. Esto redunda en su desarrollo humano, entendido como un proceso de expansión de libertades del que disfrutan los individuos en la elección de una amplia gama de oportunidades (Sen, 2000).

Frente a esta categoría, y en relación con la propuesta de desarrollo humano de Amartya Sen (2000), se reconoce que las posibilidades de apertura a nuevas oportunidades también están mediadas por la construcción colectiva frente a la adversidad, lo que Sánchez (2002) presenta como energía social y energía cultural, que define como fuentes de motivación, cohesión, persistencia humana y acción colectiva, y que en los discursos legos de Colombia se traduce como la búsqueda colectiva para salir de la vulneración constante a partir del dolor de patria, del dolor colectivo y empático. Entonces, juega 
un rol importante en la resiliencia de las mujeres víctimas el reconocimiento que provea el entorno de la colectividad como construcción social.

\section{Categoría Estado para mí. En contraste} a lo evidenciado previamente, el aporte del Estado al proceso resiliente de las mujeres participantes fue percibido como insuficiente frente a sus necesidades, dada la poca efectividad de los programas en las medidas de asistencia, atención, reparación, rehabilitación y garantías de no repetición. Tales programas son competencia del Gobierno Nacional, de acuerdo a la Ley 1448 de 2011 (Congreso de la República, 2011). Si bien se identificó que algunas medidas de asistencia humanitaria inmediata que provee el Distrito Capital son facilitadoras del proceso de resiliencia en la satisfacción de necesidades básicas, se hace hincapié, de acuerdo a la narrativa de las mujeres, en que se deben potenciar los programas de acompañamiento psicosocial con el fin de facilitar estrategias de agenciamiento y autonomía frente a la transformación de sus proyectos de vida, ocasionada por la guerra, y que a su vez interfiere en el desarrollo sostenible del país. El Estado se convierte de este modo en un medio de control para la población, la cual se ve instrumentalizada a través de políticas asistencialistas enmascaradas en ayudas económicas que no apuntan a las necesidades reales de autonomía y gobierno sobre sus propias vidas. En este sentido, las mujeres afirmaban: "no, no me han tenido en cuenta porque a nosotros a ninguno nos han tenido en cuenta nunca, apenas cuando necesitan firmas de nosotros, ahí sí nos llaman. Ahí sí me conocen que yo me llamo Z" y "capacitación porque hay gente que no tiene ninguna formación y hay gente que no sabe desempeñarse en algo, entonces pues, en un sitio donde no lo conocen... si es como algo que ayuda más".

\section{Aporte de la corporalidad frente a las estrategias de resiliencia}

Es relevante analizar las formas de indagación que se utilizaron para identificar las estrategias de resiliencia de las mujeres víctimas ante la transformación de la ocupación humana. A través de estos medios se logró reconocer que las mujeres participantes sentían mayor fluidez con la expresión verbal que con la corporal, lo cual puede entenderse como producto de que el contexto cultural ha priorizado la comunicación a través del lenguaje hablado, dejando en segundo plano aspectos corporales. Si bien el lenguaje verbal jugó un rol importante en la comprensión e identificación entre las participantes, y en el reconocimiento de las estrategias de resiliencia, el lenguaje corporal hizo posible un contacto más cercano entre ellas, la expresión de emociones y sentimientos y la transformación de su narrativa en un código meta-representacional que les permitió una exploración más profunda y personal de sí mismas y de las otras en torno a su sentir, sus sueños, sus proyectos, su misión y su fuerza motivadora en el tránsito por la ciudad. La indagación corporal permitió, no solamente identificar la resiliencia, también dio cabida al trámite emocional, generando mecanismos propios de afrontamiento y re-significación. Este trámite emocional, sumado a la construcción colectiva del código corporal, facilitó la apropiación y el 
fortalecimiento de las estrategias de resiliencia, el sentirse agentes en su propio mundo.

\section{Conclusiones}

Las principales estrategias de resiliencia frente a la transformación de actividades significativas en las mujeres participantes están relacionadas con el hacer por sus vínculos y el auto reconocimiento de capacidades y recursos propios para hacer frente a las situaciones de adversidad. Ello fortalece su propósito de "salir adelante" desde los sueños y metas personales de reivindicación de su lugar en el mundo, de trabajo por solidaridad a la sociedad, de independencia y autosuficiencia, que además se refleja en la comprensión de los hechos victimizantes como experiencia de aprendizaje y arraigo a la vida.

La percepción de las participantes frente a su capacidad de agenciamiento en el desarrollo de actividades significativas está relacionada con la independencia emocional y económica para la reconstrucción de su proyecto de vida en la ciudad, mediado a través del equilibrio emocional que les puede facilitar el medio en el que se desenvuelven. Dicho medio puede ser transformador de sus actividades cotidianas, lo que conlleva un cambio y resignificación de su ocupación humana.

La transformación de las actividades significativas, a partir de las estrategias de resiliencia que tienen las mujeres participantes, guarda relación con la búsqueda activa de nuevos escenarios de encuentro per- sonal, de reconocimiento de sus capacidades y potencialidades a partir de espacios de formación, de disfrute, de interacción con los otros y, sobre todo, de su productividad. Esto en tanto reconocen que desde que los hechos adversos ocurrieron, sus actividades significativas se han visto afectadas por el desarraigo, la inseguridad, el temor por sus vidas, la ruptura de vínculos afectivos, el castigo a la organización social y, en general, el cambio de estatus de habitante de un territorio expulsor a desplazada en la ciudad; con lo cual han desarrollado una intención de contraste que les genera bienestar y les permite resignificar el desarrollo de actividades y la participación en diferentes contextos, desde la fuerza que reflejan para hacer frente a la adversidad a partir de nuevos estilos de vida en la ciudad.

La indagación de estrategias de resiliencia a través del lenguaje corporal permitió a las investigadoras obtener evidencias para cada una de las categorías propuestas, convirtiéndose en una opción para aproximarse a la subjetividad, desde el ser y el sentir propio, y para favorecer la construcción colectiva.

Para próximas investigaciones, se sugiere realizar un abordaje con mayor número de sesiones en las que se utilicen elementos de danza que favorezcan un espacio terapéutico y potencien el proceso resiliente. Se sugiere adicionalmente indagar formas resilientes en mujeres víctimas de hechos diferentes al desplazamiento forzado que presentan una alta prevalencia y pueden incidir de manera significativa en la transformación de la ocupación humana de las mujeres, 
por ejemplo, los delitos contra la integridad sexual. Por último, se insta a indagar en las formas resilientes de mujeres en los diferentes territorios de acogida, ya que los aspectos culturales y las políticas locales influyen en su proceso de conformación.

\section{Referencias}

American Occupational Therapy Association - AOTA. (2010). Marco de Trabajo para la Práctica de Terapia Ocupacional: Dominio y Proceso. 2da edición. (Adriana Ávila, et al., trad.). Terapia-Ocupacional.com. Disponible en http://www.terapia-ocupacional.com/ aota2010esp.pdf (Obra original publicada en 2008).

Britto, D. (2009). Mujeres: el cuerpo de la Memoria. En CNRR, Grupo de Memoria Histórica, Memorias en Tiempo de Guerra. Repertorio de Iniciativas (pp.5065). Bogotá: Punto Aparte. Disponible en https://www.centrodememoriahistorica.gov.co/descargas/informes2009/ memoria_tiempos_guerra_baja.pdf

Campero, R. (2011). Los cuerpos de la Violencia. Pánico en Crisis, 3, 16-22. Disponible en http://contrapsicologia. blogspot.com.co/2011/05/revista-panico-en-crisis-n3-genero.html

Castañeda, M. (2011). El cuerpo grita lo que la boca calla. Razón y Palabra, 77 (agosto-octubre). Disponible en http://www.razonypalabra.org.mx/varia/77\%205a\%20parte/61_Castaneda_ V77.pdf

Castañeda, N. (2002). Resistencia desde la Espiritualidad. El caso de Bojayá. En otras palabras. Mujeres resistencias e irreverencias. 11 (julio-diciembre). 6367. Disponible en: http://www.bdigital.unal.edu.co/47653/1/Laresistenciadesde.pdf

Congreso de Colombia. (4 de diciembre de 2008). Ley 1257 de 2008. Por la cual se dictan normas de sensibilización, prevención y sanción de formas de violencia y discriminación contra las mujeres, se reforman los Códigos Penal, de Procedimiento Penal, la Ley 294 de 1996 y se dictan otras disposiciones. DO: 47193

Congreso de la República. (10 de junio de 2011). Ley 1448 de 2011, Por la cual se dictan medidas de atención, asistencia y reparación integral a las víctimas del conflicto armado interno y se dictan otras disposiciones. DO: 48096.

Corte Constitucional, República de Colombia. (2008). Auto 092 de 2008. Disponible en http://www.corteconstitucional.gov.co/relatoria/autos/2008/ a092-08.htm

Cyrulnik, B. (2007). De cuerpo y alma. Barcelona: Editorial Gedisa.

Fores, A. \& Grané, J. (2011). La Resiliencia: Crecer desde la adversidad. Barcelona: Plataforma Editorial.

Grupo de Investigación Ocupación y Realización Humana. (2011). Ocupación: sentido, realización y libertad. Diálogos ocupacionales en torno al sujeto, la sociedad y el medio ambiente. Bogotá: Universidad Nacional de Colombia.

Grupo de Trabajo Mujer y Género, por la Verdad, la Justicia, la Reparación y la Reconciliación. (2008). Recomendaciones para garantizar los derechos a la 
verdad, la justicia y la reparación de las mujeres víctimas del conflicto armado en Colombia. Bogotá: Ántropos. Disponible en http://www.humanas.org.co/ archivos/Recomendaciones_para_garantizar_los_derechos.pdf

Lagarde, M. (2004). Mujeres cuidadoras: entre la obligación y la satisfacción. En A. Rincón, Congreso Internacional Sare 2003: Cuidar cuesta: costes y beneficios del cuidado (pp. 155-160). Bilbao: EMAKUNDE. Disponible en: http:// www.emakunde.euskadi.eus/contenidos/informacion/pub_jornadas/es_ emakunde/adjuntos/sare2003_es.pdf

Menacho, M. (mayo, 2008). El potencial crítico de la danza contacto en la construcción de subjetividad. En Jornadas de Cuerpo y Cultura de la UNLP Universidad Nacional de La Plata, La Plata, Argentina. Disponible en http://www. memoria.fahce.unlp.edu.ar/trab_eventos/ev.673/ev.673.pdf

Muntanyola-Saura, D. \& Belli, S. (2014). Emociones y Música en movimiento. Discursos cruzados en una compañía de danza. Trans, 18, 1-27. Disponible en http://www.sibetrans.com/trans/public/docs/16-trans-2014_1.pdf

Quiroga, A. (2012) El teatro danza de Pina Bausch [En línea]. VIII Congreso Internacional de Teoría y Crítica Literaria Orbis Tertius, 7 al 9 de mayo de 2012, La Plata. En Memoria Académica. Disponible en: http://www.memoria.fahce.unlp.edu.ar/trab_eventos/ev.2569/ ev.2569.pdf
Sánchez, E. (2002) La energía social y la energía cultural, fuentes renovadoras para la acción colectiva frente a la adversidad y a las penurias. En A. Delgado (Comp.) La Resiliencia. Desvictimizar la víctima (pp. 123-140). Cali: Rafue.

Sen, A. (2000). Desarrollo y Libertad. Barcelona: Planeta.

Soler, J. (2008). Butoh (1959-2009). Medio siglo de rebelión en la danza. Acotaciones: Revista de Investigación Teatral, 20, 1-27. Disponible en http://www. resad.es/acotaciones/acotaciones20/ 20soler.pdf

Trejos, L. (2013). Colombia: Una Revisión Teórica de su Conflicto Armado. Revista Enfoques: Ciencia Política y Administración Pública, 11 (18), 55-75. Disponible en http://www.revistaenfoques.cl/index.php/revista-uno/article/ view/52/34

Valencia, (2006). La economía frente al conflicto armado interno colombiano, 1990-2006. Perfil de Coyuntura EConómica, 8 (diciembre), 141-174. Disponible en http://aprendeenlinea.udea. edu.co/revistas/index.php/coyuntura/ article/view/2321/1893 